SELÇUK ÜNIVERSITESİ

HUKUK FAKÜLTESI DERGISI

Selçuk Law Review

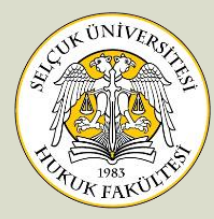

Araştırma Makalesi

Research Article

Gönderim | Received:29.04.2021

Kabul | Accepted:20.09.2021

$10.15337 /$ suhfd 929995

\title{
ANALYSE DER URTEILE DES BayObLG UND BVerfG ZUM THEMA ,DIEBSTAHL: CONTAINERN"
}

\section{Can Eralp ELİBOL*}

\section{Zusammenfassung}

Das Eigentum wird als Grundrecht im Art. 14 GG gewährleistet. Darüber hinaus hat das Eigentum auch privat- und strafrechtlichen Schutz. Diese beiden Rechtsdisziplinen sind in manchen Fällen miteinander eng verbunden. Wenn der Diebstahlverbrechen in Frage kommt, müssen manche Begriffe dieses Delikts, namentlich die Begriffe hinsichtlich des Eigentums, i.R.v. bürgerlichen Recht betrachtet werden. Nach $\S 242$ StGB wird bestraft, ,"wer eine fremde bewegliche Sache einem anderen wegnimmt". In diesem Zusammenhang ist der sog. Containern-Fall ein signifikantes Beispiel des Diebstahls. In diesem Fall geht es um die verdorbenen Lebensmittel, die auf den Abfallcontainer wegwirft und verschlossen werden, zur Abholung vom Entsorgungsunternehmen. Der Dreh- und Angelpunkt dieses Falls ist es, ob das Merkmal „Fremdheit” i.S.v. § 242 StGB hier vorliegt. Um dies festzustellen kommt $\S 959$ BGB, nämlich die Dereliktionsklausel, in Frage. Dieser Beitrag konzentriert sich auf die Betrachtung der Entscheidungen des BayObLG und BVerfG über den Containern-Fall.

Arş. Gör., Türk-Alman Üniversitesi, Hukuk Fakültesi, Ceza ve Ceza Muhakemesi Hukuku Anabilim Dalı, İstanbul, Türkiye | Asst., Turkish-German University, Law Faculty, Department of Criminal and Criminal Procedure Law, İstanbul, Turkey.

๑ can.elibol@tau.edu.tr • ORCID 0000-0001-5866-2224

** Arş. Gör., İstanbul Üniversitesi, Hukuk Fakültesi, Karşılaştırmalı Hukuku Anabilim Dalı, İstanbul, Türkiye | Asst., Istanbul University, Law Faculty, Department of Comparative Law, İstanbul, Turkey.

凹eba@istanbul.edu.tr • ORCID 0000-0003-4856-9733

Atıf Şekli | Cite As: ELIBOL Can Eralp / AKKANAT ÖZTÜRK Elif Beyza, "Analyse der Urteile des BayObLG und BVerfG zum Thema: Diebstahl: Containern", SÜHFD., C. 29, S. 4,2021 , s. 2805-2820.

İntihal | Plagiarism: Bu makale intihal programında taranmıs ve en az iki hakem incelemesinden geçmiştir. I This article has been scanned via a plagiarism software and reviewed by at least two referees. 


\section{Stichwörter}

Containern-Fall • Eigentumsaufgabe/Dereliktion $\bullet$ fremde Sache $\bullet$ Eigentum • Diebstahl

\section{BAVYERA EYALETI YÜKSEK MAHKEMESİ VE FEDERAL ALMANYA ANAYASA MAHKEMESI'NİN “HIRSIZLIK: MARKETINN ATIK BESİN MADDELERINII ÇALMAK" KONULU KARARLARININ DEĞERLENDİRILMESİ}

Öz

Mülkiyet, Alman Anayasası m. 14'te temel hak olarak korunmaktadır. Buna ek olarak mülkiyet aynı zamanda özel hukuk ve ceza hukuku korumasına da sahiptir. Bu iki hukuk disiplini bazı hâllerde birbirine sıkı sıkıya bağlıdır. Hırsızlık suçu söz konusu olduğunda bu suçun bazı kavramları, bilhassa mülkiyet ile ilişkili olanlar, medeni hukuk çerçevesinde değerlendirilmelidir. Alman Ceza Kanunu m. 242 uyarınca "her kim başkasına ait taşınır bir malı alırsa" cezalandırılır. Bu bağlamda marketin imha edilmek üzere çöp kutusuna attığ1 tüketilemez besin maddelerinin çalınması olayı, hırsızlık suçunun önemli bir örneğidir. Bu olay, bozulmuş besin maddelerinin imha edilmek üzere alınması için çöp kutusuna atılıp kilitlenerek hazır tutulması ile ilgilidir. Bu olayın can alıcı noktası, burada Alman Ceza Kanunu m. 242 anlaminda "yabancilık" unsurunun söz konusu olup olmadığıdır. Bunu tespit edebilmek için Alman Medeni Kanunu m. 959, bir diğer deyişle mülkiyetin terki hükmü gündeme gelmektedir. Bu çalışma, söz konusu olay ile ilgili Bavyera Eyaleti Yüksek Mahkemesi ve Federal Almanya Anayasa Mahkemesi'nin verdiği kararların değerlendirilmesine odaklanmaktadır.

\section{Anahtar Kelimeler}

Marketin atık besin maddelerinin çalınması olayı • Mülkiyetin terki • Başkasına ait mal• Mülkiyet • Hırsızlık 


\section{EINFÜHRUNG}

Das Eigentumsrecht ist einer von wichtigsten Grundrechten, die dem liberal demokratischen Rechtsstaat zugrunde liegt. Denn das Eigentum hat enge Verbindung mit der Freiheit ${ }^{1}$. Zudem kann die Aussage "Ohne Eigentum keine Freiheit" zitiert werden, um die Wichtigkeit dieses Rechts $\mathrm{zu}$ betonen ${ }^{2}$. Sowohl die individuelle Selbstverwirklichung als auch die Individualfreiheit richtet das Eigentumsrecht ein ${ }^{3}$. Aus diesem Grund muss es betrachtet werden, dass das Eigentum nicht nur mit der Rechtswissenschaft begrenzt werden kann. Sondern hat es zahlreiche Funktionen, und das führt uns zum Ergebnis, dass die Rechtswissenschaft diese interdisziplinären Dimensionen in Rücksicht nehmen sollte. $\mathrm{Zu}$ nennen ist es, dass das Eigentumsrecht vom höchst rechtlichen Schutz profitieren sollte. Daher hat das Eigentum als Grundrecht unter dem Art. 14 GG gewährleistet. Solches existentielle Recht braucht auch den strafrechtlichen Schutz. Deshalb fasst der Gesetzgeber unterschiedliche Delikte, die das Eigentum als Rechtsgut schützen. Einer von diesen Delikten ist der Diebstahl . Obwohl der Diebstahl als Delikt im StGB §§ 242-245 formuliert wird, verweisen diese Paragraphen auf einige Fachbegriffe, die Bedarf an der interdisziplinären Betrachtung haben. In diesem Sinn

1 PAPIER, Hans-Jürgen/SHIRVANI, Foroud, “Art. 14 [Eigentumsgarantie und Erbrecht]", Maunz/Dürig Grundgesetz Kommentar: Band II, 92. Lieferung, München, 2020, Art. 14 GG Rn. 1.

2 PAPIER/SHIRVANI, Art. 14 GG Rn. 2.

3 PAPIER/SHIRVANI, Art. 14 GG Rn. 2.

4 Das geschützte Rechtsgut des Diebstahls ist umstritten. Einer Ansicht nach ist das Eigentum allein geschütztes Rechtsgut (Siehe: WESSELS, Johannes/HILLENKAMP, Thomas/SCHUHR, Jan C., Strafrecht Besonderer Teil 2: Straftaten gegen Vermögenswerte, 42., neu bearbeitete Auflage, Heidelberg, 2019, § 2 Rn. 70; EISELE, Jörg/HEINRICH, Bernd, Strafrecht Besonderer Teil für Studienanfänger, Stuttgart, 2020, Rn. 899; KRETSCHMER, Joachim, “Diebstahl und Unterschlagung”, Strafrecht Besonderer Teil, Hrsg. HOFFMANN-HOLLAND, Klaus, Tübingen, 2015, Rn. 769; KINDHÄUSER, Urs/BÖSE, Martin, Strafrecht Besonderer Teil: Straftaten gegen Vermögensrechte, 11. Auflage, Baden-Baden, 2021, § 2 Rn. 5.). Anderer Ansicht nach schützt der Diebstahl sowohl das Eigentum als auch den Gewahrsam (Siehe: RENGIER, Rudolf, Strafrecht Besonderer Teil I: Vermögensdelikte, 16., neu bearbeitete Auflage, München, 2014, § 2 Rn. 1; SCHRAMM, Edward, Strafrecht Besonderer Teil II: Eigentums- und Vermögensdelikte, 2. Auflage, Baden-Baden, 2021, § 2 Rn. 6.). 
kommt häufig und besonders das Zivilrecht bzw. Sachenrecht ${ }^{5}$. Die Auslegung dieser Begriffe hängt nah von den Umständen einzelner Fälle ab.

Wenn der Diebstahl in Betracht kommt, fallen die Fälle hinsichtlich des Containers ein, weil die wahrscheinlich meistdiskutierten Fälle des Diebstahls sind. Diese Fälle erregen Aufsehen sowohl in der Rechtspraxis als auch in der Politik ${ }^{6}$. In diesen Fällen geht es um unverkäufliche Lebensmittel, die auf verschlossene Müllcontainern des Markts einwirft, sodass eine Entsorgungsfirma diese Lebensmittel abholen kann. Bevor die unverkäuflichen und zur Entsorgung bestimmten und bereitgestellten Lebensmittel abgeholt werden, werden sie gestohlen, indem das Schloss des Abfallcontainers aufgebrochen wird.

Die Strafgerichte verurteilten, dass sich die Täter wegen des Diebstahls in diesem sog. Containern-Fall strafbar gemacht haben. Dabei richteten die Beschwerdeführer die Verfassungsbeschwerde ein. Aber es blieb erfolglos, weil die Urteile der Strafgerichte verfassungskonform waren.

\section{RECHTSPROBLEME}

Der Inhalt dieser Fälle hatte einige Rechtsprobleme, die in den Entscheidungen diskutiert wurden. Die Probleme haben strafrechtliche und zivilrechtliche Dimensionen ${ }^{7}$. Deswegen mussten die Gerichte auf diese zwei Rechtsgebiete Rücksicht nehmen.

„Bewegliche Sache“ ist gem. § 242 StGB ein Tatbestandsmerkmal des Diebstahls. Dies enthält zwei Fachbegriffe, nämlich die Beweglichkeit und Sache. Erstens gehören alle körperlichen Gegenstände gem. § 90 BGB zum Begriff „Sache“. In diesem Zusammenhang spielt der wirtschaftliche Wert der Sache zur Tatbestandserfüllung keine Rolle ${ }^{8}$. Zweitens versteht

EISELE/HEINRICH, Rn. 897.

6 Siehe dazu: JANISCH, Wolfgang, “Containern, ein gut gemeinter Diebstahl”, Süddeutsche Zeitung, https://www.sueddeutsche.de/politik/containern-bundesverfassungsgericht-diebstahl-1.4672444, (17.04.2021); Nachrichten der beck-aktuell-Redaktion, “Experten uneinig: Soll „Containern” entkriminialisiert werden?”, becklink 2018323, beck-online, (17.04.2021).

7 KRETSCHMER, Rn. 771.

8 WESSELS/HILLENKAMP/SCHUHR, § 2 Rn. 74. 
man aus dem Adjektiv „beweglich“ eine körperliche Sache, die auch fortbewegt ${ }^{9}$, d.h. die Sache muss von einem Ort zum anderen bewegbar $\operatorname{sein}^{10}$. Auf diese Art ist erstes Problem der Gerichte, ob die unverkäuflichen Lebensmittel im Containern-Fall eine bewegliche Sache ist oder nicht.

Anderes Tatbestandsmerkmal des Diebstahls ist "die Fremdheit". Gem. § 242 StGB muss eine bewegliche Sache auch fremd sein. Die Fremdheit der beweglichen Sache kann nur im Hinblick auf die zivilrechtlichen Normen geklärt werden ${ }^{11}$, denn es gibt keine strafrechtliche Eigentumsbegriff $^{12}$. Auf diese Weise müssen die Gerichte insbesondere $\S \S 958 \mathrm{ff}$. BGB berücksichtigen. In diesem Sinne ist eine bewegliche Sache fremd, wenn es nicht allein zu dem Täter gehört oder nicht herrenlos ist ${ }^{13}$. Im sog. Containern-Fall steht die Aufgabe des Eigentums gem. §959 BGB im Vordergrund. Im §959 BGB fasste der Gesetzgeber „Aufgabe des Eigentums“ bzw. „Dereliktion“ ab. Gem. § 959 BGB „Eine bewegliche Sache wird herrenlos, wenn der Eigentümer in der Absicht, auf das Eigentum zu verzichten, den Besitz der Sache aufgibt.". Aus diesem Paragraph können zwei Begriffe "herrenlos" und "Absicht" herausgezogen werden. Herrenlos ist es, wenn eine bewegliche Sache zum Niemand gehört ${ }^{14}$, und die deutlich erkennbare Absicht des Eigentümers für Dritte spielt hier eine große Rolle. Wenn der Eigentümer der beweglichen Sache darauf Verzicht erklärt, wird die Sache als herrenlos genannt. Daher ist die Sache von diesem Zeitpunkt an nicht mehr fremd. Auf diese Art müssen die Gerichte zweitens

9 RENGIER, § 2 Rn. 5; Ob das bürgerlichen Recht als Orientierungspunkt genommen werden muss, gehen die Meinungen auseinander. Siehe dazu: WESSELS/HILLENKAMP/SCHUHR, § 2 Rn. 78.

10 ARZT, Gunther/WEBER, Ulrich/HEINRICH, Bernd/HILGENDORF, Eric, Strafrecht Besonderer Teil, 2. Auflage, Bielefeld, 2009, § 13 Rn. 32.

11 RENGIER, § 2 Rn. 6; EISELE/HEINRICH, Rn. 909; ARZT/WEBER/HEINRICH/HILGENDORF, § 13 Rn. 33; WITTIG, Petra, "Diebstahl und Unterschlagung", BeckOK StGB, Hrsg. von HEINTSCHEL-HEINEGG, Bernd, 49. Edition, München, 2021, § 242 StGB Rn. 6; KINDHÄUSER, Urs, "Diebstahl und Unterschlagung", Kindhäuser/Neumann/Paeffgen Strafgesetzbuch: Band 1, 5. Auflage, Baden-Baden, 2017, § 242 StGB Rn. 15.

12 BOSCH, Nikalaus, "Diebstahl und Unterschlagung", Schönke/Schröder Strafgesetzbuch: Kommentar, 30., neubearbeitete Auflage, München, 2019, § 242 StGB Rn. 12.

13 KRETSCHMER, Rn. 774; SCHRAMM, § 2 Rn. 16.

$14 \quad$ KINDHÄUSER/BÖSE, § 2 Rn. 16. 
prüfen, ob die unverkäuflichen Lebensmittel, die auf den Müllcontainer geworfen haben, das Merkmal „fremd" darstellen zu können.

\section{ERGEBNISSE UND BEGRÜNDUNG DES BayObLG}

Bayerische Oberlandesgericht (BayObLG) fand die Feststellungen des Amtsgerichts (AG), die die Lebensmittel aus dem abgesperrten Container im Firmengelände weggenommen wurden, rechtmäßig. Daher begegnete diese Annahme vom AG keinen rechtlichen Bedenken ${ }^{15}$.

Nach der Entscheidung des BayObLG kommen bewegliche Sachen in Frage, da die unverkäuflichen Lebensmittel körperlich und fortbewegt sind. Die Fremdheit der Sachen aber hängt von den Umständen des Einzelfalles ab, weil das Wegwerfen nicht immer der Abgabe des Eigentums verursacht ${ }^{16}$. Das Verhalten des Eigentümers muss daher für Dritte deutlich erkennbar sein. In diesem Fall entschied das BayObLG, dass die Dereliktion bzw. Eigentumsaufgabe hier nicht vorlag17. Denn der Container war verschlossen und stand auf dem Grundstück des Supermarkts bereit zur Abholung durch das Entsorgungsunternehmen.

Eine Dereliktion liegt dann vor, wenn der Eigentümer in der Absicht, die Sachen aufgeben zu wollen. In diesem Fall wollte der Supermarkt das Eigentum der nicht verkehrsfähigen Lebensmittel nicht abgeben, sondern wollte er die Lebensmittel zugunsten dem Entsorgungsunternehmen abgeben. Außerdem machte er seine Absicht deutlich erkennbar, da der verschlossene Container im Zulieferbereich des Supermarkts stand. Außerdem hatte der Supermarkt die Verantwortung, die Abfälle entsorgen zu lassen ${ }^{18}$. Aus diesem Grund blieben die Lebensmittel bis zur Abholung der Lebensmittel vom Entsorgungsunternehmen im Eigentum des Supermarkts.

Die Wertlosigkeit der Lebensmittel kann nicht die Wegnahme dieser Lebensmittel rechtfertigen, weil auch "geringwertige Sache" gem. § 243 Abs. 2 StGB das Tatobjekt des Diebstahls sein kann.

\footnotetext{
15 NStZ-RR 2020, 104, beck-online, S. 104.

16 NStZ-RR 2020, 104, beck-online, S. 104.

17 NStZ-RR 2020, 104, beck-online, S. 105.

18 NStZ-RR 2020, 104, beck-online, S. 105.
} 


\section{DIE ENTSCHEIDUNG DES BVerfG ZUM THEMA „DIEB- STAHL: CONTAINERN"}

Nachdem das BayObLG die Revisionen der Täter im ContainernFall unbegründet gefunden hatte, legten die Täter dagegen eine Verfassungsbeschwerde ein. Die Beschwerdeführer (Bf.) beanspruchten, dass die Entscheidungen der Strafgerichte ihr allgemeines Persönlichkeitsrecht i.S.v. Art. 2 I GG i.V.m. Art. 1 I GG und ihre allgemeine Handlungsfreiheit i.S.v. Art 2 I GG verletzten.

Nach der Entscheidung des BVerfG haben die Fachgerichte die Obliegenheit, die Umstände der Einzelfälle zu bewerten. Im vorliegenden Fall stellten die Fachgerichte die Voraussetzungen der Eigentumsaufgabe nach $\S 959$ BGB dar und sagte, dass der Müllcontainer in der Anlieferzone des Supermarkts stand und abgesperrt war. Außerdem wurde der Abfallcontainer zur Abholung des Entsorgungsunternehmens bereitgestellt. Diese Feststellungen verursachten dem Ergebnis, dass kein Verzichtswille in diesem Fall festgelegt werden konnte. In Bezug auf diese Feststellungen der Fachgerichte erkannte das BVerfG keine Rechtsfehler und daher lag hier kein Verstoß gegen das Verfassungsrecht vor ${ }^{19}$. Außerdem ist diese zivilrechtliche Auslegung der Fremdheit von den Fachgerichten im Rahmen des $\S 242$ StGB nicht willkürlich, weil solche Interpretation mit der Rechtseinheit und Rechtssicherheit in Einklang ist ${ }^{20}$.

Unter dem Art. 2 I GG wurde die Handlungsfreiheit gewährleistet. Der folgende Teil des Art. 2 I GG beschränkt die Handlungsfreiheit. Die Handlungsfreiheit darf beschränkt werden, falls der Person "die Rechte anderer verletzt und gegen die verfassungsmäßige Ordnung oder das Sittengesetzt verstößt". Laut der Entscheidung des BVerfG darf BVerfG nur die Verfassungsmäßigkeit der Strafvorschriften prüfen ${ }^{21}$. Das BVerfG darf nicht die Sache des Gesetzgebers bestimmen. Unter dem Art. 14 I GG gewährleistetes Eigentum hat große Bedeutung für den sozialen Rechtstaat und daher genießt es den besonderen Schutz, es sei denn, die Sicherung der persönlichen Freiheit ist nicht vorhanden ${ }^{22}$. Auf diese Art ist $\S 242$

\footnotetext{
19 NJW 2020, 2953, beck-online, Rn. 29.

$20 \quad$ NJW 2020, 2953, beck-online, Rn. 25.

$21 \quad$ NJW 2020, 2953, beck-online, Rn. 37.

22 NJW 2020, 2953, beck-online, Rn. 39.
} 
StGB und daraus resultierende Strafbarkeit der Wegnahme von den Lebensmitteln in diesem Fall kein Verstoß gegen die Handlungsfreiheit, weil der Schutz der berechtigten Rechtsposition des Eigentümers vorhanden ist und der Inhalt und die Schranken des Eigentumsrechts vom Gesetzgeber durch ein Gesetzt bestimmt werden ${ }^{23}$.

$\S 242$ StGB schützt nicht nur das Eigentumsrecht, sondern auch die Möglichkeit, die vom § 903 BGB entsteht d.h. der Eigentümer kann nach seinem Wunsch mit der Sache verfahren oder nicht ${ }^{24}$. Auf diese Art schützt $\S 242$ StGB den Eigentümer auch vom Umgang jeder Dritte mit der Sache. Davon ist der wirtschaftliche Wert der Sache unabhängig ${ }^{25}$. Deshalb liegt hier kein Verstoß gegen das Ultima-Ratio-Prinzip i.S.v. der Verhältnismäßigkeit wegen der Strafbarkeit des Diebstahls vom Container vor ${ }^{26}$.

In diesem Fall richtete das Verhalten der Bf. auf dem Grundrecht „Eigentum“ gem. Art. $14 \mathrm{GG}^{27}$. Allerdings wurde hier nicht rein formale und inhaltsleere Eigentumsposition geschützt. Im Gegensatz dazu wurde das legitime Interesse der Eigentümer, nämlich die Entsorgung der Lebensmittel hinsichtlich ihrer Sicherheit, im vorliegenden Fall geschützt ${ }^{28}$. Da der Gesetzgeber auch die geringere Schuld des Täters im Rahmen des $\S 242$ StGB strafbar gemacht hat, stand die Strafbarkeit der Bf. im vorliegenden Fall in Einklang mit dem GG und daher war die Entscheidung der Fachgerichte verhältnismäßig ${ }^{29}$. Vielmehr gibt es zahlreiche Normen im allgemeinen Teil des StGB, die die Gerichte ermöglichen, um die Strafe bezüglich der Schuld des Täters und unter den Umständen des Einzelfalls zu bemessen ${ }^{30}$. Im vorliegenden Fall berücksichtigten die Fachgerichte bei der Strafbemessung die Besonderheiten des Falls, aus diesem Grund liegt hier kein Verstoß gegen das Verfassungsrecht vor ${ }^{31}$.

\footnotetext{
23 NJW 2020, 2953, beck-online, Rn. 39, 40.

24 NJW 2020, 2953, beck-online, Rn. 40.

25 NJW 2020, 2953, beck-online, Rn. 40.

$26 \quad$ NJW 2020, 2953, beck-online, Rn. 32.

$27 \quad$ NJW 2020, 2953, beck-online, Rn. 42.

28 NJW 2020, 2953, beck-online, Rn. 42.

29 NJW 2020, 2953, beck-online, Rn. 45.

30 Bsp. $\S \S 47,56,59,60$ StGB; NJW 2020, 2953, beck-online, Rn. 46.

$31 \quad$ NJW 2020, 2953, beck-online, Rn. 47.
} 


\section{LITERARISCHES ECHO}

Auch im Schrifttum erregt das Thema Aufsehen. Der Schlüssel dieses Falls ist nach § 959 BGB geregelte Dereliktion. Ob die verdorbenen Lebensmittel im Container ein taugliches Tatobjekt sind, müssen der Wille des Eigentümers und alle Besonderheiten des Falls betrachtet werden $^{32}$. Zuerst wird es festgelegt, dass die Lebensmittel als körperliche Gegenstände beweglich sind ${ }^{33}$. Als Zweite sollte die Fremdheit der Lebensmittel diskutiert werden. Nach Rengier kommt die Dereliktionsabsicht nicht in Frage, wenn die Sache auf die Mülltonne geworfen wird, um sie vernichten zu lassen ${ }^{34}$. Eisele/Heinrich zufolge ist es ausschlaggebend, dass der Verwendungszweck des Eigentümers für Dritte deutlich erkennbar ist $^{35}$. In diesen Fällen sind gestohlene Lebensmittel nicht herrenlos, weil der Supermarkt das Eigentum behält, bis das Entsorgungsunternehmen sie abholt ${ }^{36}$. Daher liegt hier dann das Merkmal "fremd“ nicht vor. Laut Schramm, Esser/Scharnberg und Wittig sollte die Verschlossenheit des Containers zugrunde legen, denn solange der Container abgeschlossen ist, steht die Verzichtabsicht des Eigentümers nicht im Vordergrund ${ }^{37}$. AuBerdem spielt wirtschaftlicher Wert der Lebensmittel keine Rolle, um den Täter vom Diebstahl strafbar machen zu können ${ }^{38}$. Für die Entkleidung der Fremdheit der solchen Lebensmittel sollen neue zivilrechtliche Regelungen brauchen, um die Firmen unverkäufliche Lebensmittel zur Spende zu verpflichten ${ }^{39}$.

Nach Kindhäuser/Böse können die Haftungsrisiken des Supermarkts, die aus der Verantwortung des Supermarkts von unverkäuflichen Le-

\footnotetext{
32 ESSER, Robert/SCHARNBERG, Josephine, “Anfängerklausur - Strafrecht: Containern", JuS, Heft: 9, 2012, S. 810.

33 ESSER/SCHARNBERG, S. 812.

$34 \quad$ RENGIER, § 2 Rn. 7.

35 EISELE/HEINRICH, Rn. 910; KINDHÄUSER/BÖSE, § 2 Rn. 18.

36 KRETSCHMER, Rn. 774; BOSCH, § 242 Rn. 17/18.

37 SCHRAMM, § 2 Rn. 18; ESSER/SCHARNBERG, S. 810; WITTIG, § 242 Rn. 9.3.

38 SCHRAMM, § 2 Rn. 18; BOSCH, § 242 StGB Rn. 4.

39 SCHRAMM, § 2 Rn. 18.
} 
bensmitteln entstehen mögen, zur Annahme von der Aufgabe des Eigentums nicht führen ${ }^{40}$. Im Gegenteil verursacht der Konsum der verdorbenen Lebensmittel der Abnahme vom Absatz des Supermarkts ${ }^{41}$.

$\mathrm{Ob} \S 959$ BGB obsolet ist, wird aber es verneint ${ }^{42}$. Hinzu kommt, dass sich $\S 3 \mathrm{I} \mathrm{Krw}-/ \mathrm{AbfG}^{43}$ und $\S 15 \mathrm{I} \mathrm{Krw}-/ \mathrm{AbfG}^{44}$ kein Besitzaufgabeverbot ergeben.

\section{PERSÖNLICHE MEINUNG}

\section{A. Privatrechtliche Betrachtung des Containern-Falls}

Obwohl es bei der Entscheidung darum geht, ob das Diebstahlsverbrechen stattgefunden hat oder nicht, sollte der Vorfall auch privatrechtlich behandelt werden. In diesem Zusammenhang sollte es zunächst erörtert werden, ob der Supermarkt das Eigentumsrecht an den Vermögenswerten hat, von denen behauptet wird, dass sie Gegenstand von Diebstahl seien. Es sollte erklärt werden, ob die unverkäuflichen Lebensmittel vom Marktbesitzer aufgegeben und von seinem Eigentum entfernt wurden. In diesem Fall sollte es auch offengelegt werden, ob die Elemente der Aufhebungsklausel des § 959 BGB vorhanden waren.

Die Aufgabe des Eigentums ist im § 959 BGB wie folgt definiert: „Eine bewegliche Sache wird herrenlos, wenn der Eigentümer in der Absicht, auf das Eigentum zu verzichten, den Besitz der Sache aufgibt." § 959 BGB regelt den "Eigentumsverlust durch Verzicht bei beweglichen Sachen ${ }^{45}$ “. Der mögliche Entscheidungsverlust ist ein einseitiges Rechtsgeschäft mit doppelter

40 KINDHÄUSER/BÖSE, § 2 Rn. 18.

41 KINDHÄUSER/BÖSE, § 2 Rn. 18.

42 SCHULTE-NÖLKE, Hans, "Eigentum", Bürgerliches Gesetzbuch: Handkommentar, 10. Auflage, Baden-Baden, 2019, § 959 BGB Rn. 1; BERGER, Christian, "Eigentum", Jauernig Bürgerliches Gesetzbuch: Kommentar, 18. Auflage, München, 2021, § 959 BGB Rn. 3.

43 SCHULTE-NÖLKE, § 959 BGB Rn. 1.

44 KINDHÄUSER/BÖSE, § 2 Rn. 18.

45 HOEREN, Thomas, "Aneignung", Ring/Grziwotz/Keukenschrijver, BGB Sachenrecht: Band 3, Hrsg. GRZIWOTZ, Herbert, KEUKENSCHRIJVER, Alfred, RING, Gerhard, 4. Auflage, 2016, § 959 BGB Rn. 1. 
Natur: (1) Objektiv gehören sie zur eigentlichen Eigentumsaufgabe und (2) subjektiv zum Willen, das Eigentum aufzugeben ${ }^{46}$.

Eine einseitige Absichtserklärung ist erforderlich, die keinen Erhalt erfordert, um das Eigentum am Gegenstand aufzugeben ${ }^{47}$. Diese Erklärung ist auslegungsfähig und als Absichtserklärung im Sinne der §§ 116 ff. BGB anfechtbar. Daher kann es auch impliziert werden. Die Anfechtung hinsichtlich des mangelnden Willens muss an die Person gerichtet werden, die die Sache nach dem Verzicht auf das Eigentum in Besitz genommen hat. Wenn die Sache noch nicht zurückgenommen wurde, ist eine Vermeidungserklärung nicht erforderlich. Hier reicht die bloße Rücknahme.

Ein typischer Grund zur Vermeidung ist der Fehler in Bezug auf die Eigenschaften der Sache wie der Wert oder die Benutzerfreundlichkeit ${ }^{48}$. Wenn sich der Eigentümer über die Identität der Sache geirrt hat oder es ihm nicht bewusst war, dass er objektiv das Eigentum der Sache aufgegeben hat, erfordert eine Einrede mangels der Willenserklärung nicht ${ }^{49}$. So zB der Besitzer eine vermeintlich leere Schachtel wegwirft, die noch ein Objekt enthält, oder wenn er künstlerische Skizzen anstelle von altem Papier wegwirft ${ }^{50}$. Selbst die Bereitstellung alter Kleidersäcke mit dem Label „Rotes Kreuz" macht die Kleidungsstücke nicht im Sinne der Verordnung "aufgegeben" 51 .

Auch die Absicht, eine Sache zu zerstören, impliziert nicht immer den Wille, das Eigentum aufzugeben. Beispielsweise kann im Fall vom normalen Hausmüll oder Sperrmüll der Schluss gezogen werden, dass die Absicht besteht, eine Abwertung vorzunehmen. Anders verhält es sich mit persönlichen Dokumenten, die in den Papierkorb geworfen wurden, um sie vernichten zu lassen. Wer denn beispielsweise Briefe, Bankdokumente oder Geschäftspapiere wegwirft, wollte es nicht, dass der

46 HOEREN, §959 BGB Rn. 1.

47 HOEREN, § 959 BGB Rn. 3; OECHSLER, Jürgen, “Eigentum”, Münchener Kommentar zum Bürgerlichen Gesetzbuch: Band 8, 8. Auflage, München, 2020, § 959 BGB Rn. 6.

48 HOEREN, §959 BGB Rn. 3; OECHSLER, § 959 BGB Rn. 3.

49 HOEREN, §959 BGB Rn. 4.

50 HOEREN, §959 BGB Rn. 3; OECHSLER, § 959 BGB Rn. 3.

51 HOEREN, §959 BGB Rn. 3. 
Dritte sie nimmt und möglicherweise gegen den Vorbesitzer verwendet. Hier ist der Wille von Wegwerfende nicht auf eine Aufgabe des Eigentums sondern auf eine Übertragung nach dem § 929 BGB an die Entsorgungsunternehmen gerichtet ${ }^{52}$.

Es wird argumentiert, die Absichtserklärung des Eigentümers gem. $\S 134$ nichtig wäre, wenn sie gegen ein gesetzliches Verbot des Verfalls verstieße. Nach der richtigen Ansicht enthalten die Normen wie $\S 15$ Abs. $1 \mathrm{KrWG}$ keine Verfallsverbote. § 15 Abs. 2 KrWG verbietet jedoch bestimmte Formen der Verfehlung. Wenn der Wille des Eigentümers daher auf eine verbotene Form der Aufgabe des Eigentums gerichtet hat -die Verletzung des Verbots wird durch die Art und Weise begangen, in der der Eigentümer aufgegeben wird-, muss die gem. § 959 BGB Absichtserklärung nach $\S 134$ BGB ebenfalls nichtig sein, sodass der Eigentümer auf keinen Fall aus der Verantwortung heraus entlassen wird ${ }^{53}$. Die Tatsache ist es, dass die Person gesetzlich verpflichtet ist, den betreffenden Gegenstand in Besitz zu nehmen, bedeutet es jedoch nicht, dass sie ihn nicht verlassen kann. Wenn jemand ein Eigentum aufgibt, der § 15 Abs. 2 KrWG definiert ist, wird die entsprechende Sache aus seinem Vermögen entfernt. Wenn aber die Person diese Sache aufgegeben hat, ist sie verpflichtet, den durch diese Sache verursachten Schaden zu beheben. So die Absicht des $\S 15$ Abs. 2 KrWG nicht darin besteht, die Einsparungsmöglichkeit von den Einzelpersonen einzuschränken oder einen Grund für die Ungültigkeit eines Rechtsgeschäfts anzugeben.

Schließlich müssen die Absichtserklärung und die Aufgabe der Eigentumskontrolle gleichzeitig vorliegen ${ }^{54}$.

Der Gegenstand des Besitzes ist ein Realakt. Er muss tatsächlich durchgeführt werden. Im konkreten Containern-Fall hängt die Aufgabe des Eigentums an den diebstahlgefährdeten Gegenständen daher von der Existenz dieser beiden Elemente ab:

- Als erstes muss es festgestellt werden, dass der Marktbesitzer keinen Willen zur Aufgabe hatte. Dies liegt daran, dass die unverkäuflichen

\footnotetext{
52 HOEREN, §959 BGB Rn. 4; OECHSLER, § 959 BGB Rn. 3.

53 OECHSLER, § 959 BGB Rn. 7; KINDL, Johann, "Eigentum", BeckOK BGB, Hrsg. HAU, Wolfgang und POSECK, Roman, 57. Edition, 2021, § 959 BGB Rn. 5; NJW 2018, 3125, beck-online, Rn. 13, 16.

54 OECHSLER, § 959 BGB Rn. 9.
} 
Lebensmittel in einem verschlossenen Container an einem Ort unter ihrem eigenen Eigentum aufbewahrt wurden.

- Im Gegenteil dazu: Wenn das Ereignis als Ganzes betrachtet wird, kann es ausgesagt werden, dass der Supermarkt den Willen hatte, sich in Übereinstimmung mit seinen aus dem Gesetz ergebenden Verpflichtungen handeln zu haben. Tatsächlich hat der Supermarkt auch einen Vertrag mit dem Entsorgungsunternehmen über die Entsorgung von Abfällen unterzeichnet. Nach diesem Vertrag wurden die unverkäuflichen Lebensmittel bis zur Lieferung aufbewahrt.

- Darüber hinaus unterlag die Verschwendung des Supermarkts einem Vertragsverhältnis. Damit der Supermarkt den Vertrag ordnungsgemäß erfüllen konnte, musste er unverkäufliche Lebensmittel enthalten. Die Aufgabe der unverkäuflichen Lebensmittel wäre auch gegen ihre eigenen Interessen.

- Es kann festgestellt werden, dass solcher Wille des Supermarkts im konkreten Fall nicht existierte, ohne das Argument, dass er diese unverkäuflichen Lebensmittel aufgrund seiner gesetzlichen Verpflichtung nicht aufgeben konnte.

- Infolge dieser Situation kann davon ausgegangen werden, dass in diesem Fall weder ein objektives noch ein subjektives Erfordernis der Aufgabe des Eigentums besteht.

\section{B. Strafrechtliche Betrachtung des Containern-Falls}

Das Eigentum wird in den $\S \S 242 \mathrm{ff}$. StGB durch das Strafrecht geschützt. Nach § 242 StGB: „Wer eine fremde bewegliche Sache einem anderen in der Absicht wegnimmt, die Sache sich oder einem Dritten rechtswidrig zuzueignen..." sich strafbar macht. Im Rahmen dieses Paragraphs stehen die Begriffe „Fremdheit“, „Beweglichkeit" und „Zueignungsabsicht" im Vordergrund. Im Containern-Fall sollte sich auf den Begriff „Fremdheit” fokussiert werden, da keine Diskussion über die Beweglichkeit der Lebensmittel und rechtswidrige Zueignungsabsicht von den Tätern hier vorliegt. In diesem Fall warf der Supermarkt die verdorbenen Lebensmittel auf den verschlossenen Müllcontainer weg und stellte sie zur Abholung vom Entsorgungsunternehmen bereit. Ob das Wegwerfen der Lebensmittel als Aufgabe des Eigentums gesehen werden kann, spielt hier diese Entschei- 
dung eine große Rolle, um die Fremdheit der Sache festzustellen. Hauptsächlich hat solche wichtige Entscheidung über die Eigentumsaufgabe Bedarf an zivilrechtorientierter Auslegung. Laut der obigen Erklärungen über die Aufgabe des Eigentums bzw. Dereliktion kann es ausgedrückt werden, dass der Supermarkt nach den Umständen des Falls nicht in der Absicht, das Eigentum der verdorbenen Lebensmittel aufzugeben. Sondern hatte er die Vernichtungsabsicht d.h. er wollte die Lebensmittel durch das Entsorgungsunternehmen vernichten lassen. Aus diesem Grund liegt hier keine Dereliktion i.S.v. § 959 BGB vor. Daher sind diese Lebensmittel noch fremd im Rahmen des $\S 242$ StGB. Im Endeffekt haben sich die Täter durch die tatbestandmäßige Wegnahme wegen des Diebstahls strafbar gemacht.

\section{SCHLUSS}

Das Eigentumsrecht ist einer von den bedeutsamsten und wichtigsten Grundrechten, das im Art. 14 GG gewährleistet wird. Hinzu kam, das Eigentum wird sowohl im Bereich des Strafrechts als auch Zivilrechts geschützt. Für strafrechtlichen Schutz hat der Gesetzgeber verschiedene Delikte abgefasst. In diesem Beitrag wird sich auf den Diebstahl, namentlich den Containern-Fall, fokussiert. Obwohl der Diebstahl i.S.v. §§ 242 ff. StGB in Bezug auf das Strafrecht betrachtet wird, werden einige Merkmale des Diebstahls angesichts des bürgerlichen Rechts im Rahmen des BGB interpretiert. In besonderem Maße steht hier im Hinblick auf $\S 953$ BGB ausgelegtes Straftatbestandsmerkmal "fremd“ im Vordergrund. Nach § 953 BGB spielt die von jedem Beliebigen deutlich erkennbaren Verzichtsabsicht des Eigentümers große Rolle. Im sog. Containern-Fall liegt aber keine Eigentumsaufgabe vor, weil sich die Dereliktionsabsicht aus den Umständen und Besonderheiten des Falls nicht ergeben. Wie in diesem Fall führt das Wegwerfen einer Sache nicht zum Verzichtswille. Zum Schluss ist die Wegnahme von den Lebensmitteln aus den verschlossenen Containern im Firmengelände eine tatbestandsmäßige Handlung im Rahmen des § 242 StGB und daher haben sich die Täter strafbar gemacht. 


\section{LITERATUR}

ARZT, Gunther/WEBER, Ulrich/HEINRICH, Bernd/HILGENDORF, Eric, Strafrecht Besonderer Teil, 2. Auflage, Bielefeld, 2009.

BERGER, Christian, “Eigentum", Jauernig Bürgerliches Gesetzbuch: Kommentar, 18. Auflage, München, 2021.

BOSCH, Nikalaus, "Diebstahl und Unterschlagung”, Schönke/Schröder Strafgesetzbuch: Kommentar, 30., neubearbeitete Auflage, München, 2019.

EISELE, Jörg/HEINRICH, Bernd, Strafrecht Besonderer Teil für Studienanfänger, Stuttgart, 2020.

ESSER, Robert/SCHARNBERG, Josephine, “Anfängerklausur - Strafrecht: Containern", JuS, Heft: 9, 2012, S. 809-814.

HOEREN, Thomas, "Aneignung ", Ring/Grziwotz/Keukenschrijver, BGB Sachenrecht: Band 3, Hrsg. GRZIWOTZ, Herbert, KEUKENSCHRIJVER, Alfred, RING, Gerhard, 4. Auflage, 2016.

KINDHÄUSER, Urs/BÖSE, Martin, Strafrecht Besonderer Teil: Straftaten gegen Vermögensrechte, 11. Auflage, Baden-Baden, 2021.

Nachrichten der beck-aktuell-Redaktion, “Experten uneinig: Soll „Containern” entkriminialisiert werden?", becklink 2018323, beck-online.

NJW 2018, 3125, beck-online. (Erişim Tarihi: 05.04.2021)

NJW 2020, 2953, beck-online. (Erişim Tarihi: 05.04.2021)

NStZ-RR 2020, 104, beck-online. (Erişim Tarihi: 05.04.2021)

JANISCH, Wolfgang, "Containern, ein gut gemeinter Diebstahl”, Süddeutsche Zeitung, https://www.sueddeutsche.de/politik/containern-bundesverfassungsgericht-diebstahl-1.4672444.

KINDHÄUSER, Urs, "Diebstahl und Unterschlagung", Kindhäuser/Neumann/Paeffgen Strafgesetzbuch: Band 1, 5. Auflage, Baden-Baden, 2017.

KINDL, Johann, “Eigentum”, BeckOK BGB, Hrsg. HAU, Wolfgang und POSECK, Roman, 57. Edition, 2021.

KRETSCHMER, Joachim, “Diebstahl und Unterschlagung”, Strafrecht Besonderer Teil, Hrsg. HOFFMANN-HOLLAND, Klaus, Tübingen, 2015. 
OECHSLER, Jürgen, “Eigentum”, Münchener Kommentar zum Bürgerlichen Gesetzbuch: Band 8, 8. Auflage, München, 2020.

PAPIER, Hans-Jürgen/SHIRVANI, Foroud, “Art. 14 [Eigentumsgarantie und Erbrecht]", Maunz/Dürig Grundgesetz Kommentar: Band II, 92. Lieferung, München, 2020.

RENGIER, Rudolf, Strafrecht Besonderer Teil I: Vermögensdelikte, 16., neu bearbeitete Auflage, München, 2014.

SCHRAMM, Edward, Strafrecht Besonderer Teil II: Eigentums- und Vermögensdelikte, 2. Auflage, Baden-Baden, 2021.

SCHULTE-NÖLKE, Hans, “Eigentum”, Bürgerliches Gesetzbuch: Handkommentar, 10. Auflage, Baden-Baden, 2019.

WESSELS, Johannes/HILLENKAMP, Thomas/SCHUHR, Jan C., Strafrecht Besonderer Teil 2: Straftaten gegen Vermögenswerte, 42., neu bearbeitete Auflage, Heidelberg, 2019.

WITTIG, Petra, "Diebstahl und Unterschlagung", BeckOK StGB, Hrsg. von HEINTSCHEL-HEINEGG, Bernd, 49. Edition, München, 2021. 